\title{
Introduction to the special issue on software defined radio: selected papers from the Wireless Innovation Forum's SDR'11-WinnComm
}

\author{
John Glossner • Mohammed Ismail • \\ Jarmo Takala
}

Received: 25 September 2012/ Accepted: 25 September 2012/Published online: 7 October 2012

(C) Springer Science+Business Media New York 2012

This special issue contains extended articles based on the best papers of the $R \& D$ track of the Wireless Innovation Forum's SDR'11-WinnCommConference on Wireless Communications Technologies and Software Defined Radio (SDR).

The Wireless Innovation Forum (previously known as the SDR Forum) was established in 1996. The Wireless Innovation Forum ${ }^{\mathrm{TM}}$ is a non-profit "mutual benefit corporation" dedicated to driving technology innovation in commercial, civil, and defense communications around the world. Forum members bring a broad base of experience in SDR, Cognitive Radio(CR), and Dynamic Spectrum Access (DSA) technologies in diverse markets and at all levels of the wireless value chain to address emerging wireless communications requirements through enhanced value, reduced total life cost of ownership, and accelerated deployment of standardized families of products, technologies, and services. The Forum acts as the premier venue for its members to collaborate to achieve these objectives, providing opportunities to network with customers,

\footnotetext{
J. Glossner

Optimum Semiconductor Technologies, Inc., 120 White Plains

Rd, Tarrytown, NY 10601, USA

e-mail: jglossner@optimumsemi.com

M. Ismail

Khalifa University of Science Technology and Research

(KUSTAR), Sharjah, UAE

e-mail: ismail@kustar.ac.ae

J. Takala $(\bowtie)$

Tampere University of Technology, Korkeakoulunkatu 1, 33101

Tampere, Finland

e-mail: jarmo.takala@tut.fi
}

partners and competitors, educate decision makers, develop and expand markets, and advance relevant technologies.

The Technical Conference was first established in 1992 after a highly successful workshop held in 1991. Now attracting a broad range of about 500 delegates including researchers, professors, industry developers, investors, commercial network operators, radio manufacturers, system integrators, government procurement officials, regulators, engineering service providers, and consultants from over 22 different countries, the conference is the only event devoted to the advancement of reconfigurable radio technologies from research through deployment.

We are pleased to report that the annual US conference and product exhibition continues to hold its place in the advanced wireless community as the premier event for exploring SDR, $\mathrm{CR}$, and DSA technologies. Last year there was continued strong participation in this conference. In spite of the continuing down turn in the economy in many segments of the market, our papers were downloaded more than 81,000 times.

A unique feature of this conference is in providing opportunities to network from a broad mix of participants from all levels of the wireless value chain including investors, commercial network operators, radio manufacturers, system integrators, government procurement officials, regulators, technology providers, engineering service providers, and consultants. Of this group $42 \%$ were researchers and technology developers.

From this group of researchers, academically focussed papers are submitted to the Research and Development (R\&D) Track of the conference. This year's paper topics included SDR implementations and architectures, Communications Signal Processing, Physical Layer Techniques, Chip Implementations, GPUs, FPGAs, Processors, RF Technologies, Security, Software Systems, SCA, Spectrum Sharing, CR, System Implementation, and Testing. 
This special issue specifically highlights extended versions of the best R\&D papers from the 2011 SDR'11WinnComm conference. These papers were evaluated by world experts in SDR, CR, DSA, and Communications System Design. Academically focused and reviewed, these papers represent the leading edge of research in this area.

The first four articles in this special issue are related to receiver technologies. Yin and Cavallaro consider interference cancellation in LTE. They propose a receiver using partial interface cancellation and time domain equalization. The proposed system has been implemented on FPGA and the experiments show low latency and area efficiency of the proposed approach. Ramkumar and Bose discuss multiuser modulation classification and they propose multiantenna cognitive receivers with MIMO blind equalization. The aim is to improve multiuser symbol detection and modulation classification. The performance of the receivers is verified with the aid of simulations. MIMO sphere detection is discussed by Wu, Dick, Sun, and Cavallaro. They propose a detector design where V-BLAST-type of pre-processing before detection is avoided, thus reducing the complexity. The proposed detector is scalable and shows area efficiency compared to previous designs. Base stations are considered by Marojevic, Gomez, Gilabert, Montoro, and Gelonch. They propose base stations to be implemented as SDR clouds where distributed antennas connected to a data center carry out the DSP processing. The authors analyze different resource management strategies for SDR clouds and conclude that the most flexible solution is obtained by combining several methods as a hierarchical resource management.

There are three articles on CRs. Roof and Fam consider signal classification. The authors propose a modulation detection method based on peak frequency-magnitude histogram. The method can detect multiple signals within given bandwidth and their frequency density features. The authors use decision tree classifier to illustrate performance of automatic signal classifier. This paper won the best paper award in SDR'11-WinnComm conference. Sutton and Doyle discuss detection of cyclostationary signatures embedded in multicarrier waveforms. Frequency-selective fading conditions typically destroy such signatures. The paper proposes a detector based on spectral correlation function, which is improved with spectral coherence estimation. Simulations illustrate the robustness of the detector. Spectrum sharing is the topic of article by Okamoto, Ohta, Ineke, Fujii, and Ariyoshi. They propose a channel allocation method, which uses a frequency priority table based on the location of the secondary system. Interference from other secondary sources is reduced with additional power control. The interference performance and throughput of the method is shown with the aid of simulations.

Radio architectures and physical layer technologies are the topic in three articles. Frequency hopping systems is discussed by harris, Chen, and Venosa who propose an architecture where signal hopping and de-hopping are performed in digital domain. The system is based on using polyphase up converter and down converter channelizers. The system contains also a fully digital synchronization for signal acquisition and tracking. This paper won the best paper award in SDR'11-WinnComm conference. In their article, harris, Venosa, Chen, and Renfors consider digital down converters. They propose alternate filter structures that offer significant computational advantages over the conventional down converters based on cascade integratorcomb filters. Yenamandra and Ismail discuss spectrum sensing and TV white space applications. They propose a low power spectrum sensing architecture for CRs. They assume that receiver is aware of pilot patterns from primary users. The circuit realization has been verified with the aid of simulations to meet the ECMA-392 specification.

SDR systems based on graphic processing units are considered in three articles. Yang, Kim, Ahn, Kim, Choi, and Glossner have implemented a $2 \times 2$ MIMO WiMAX system with 16-QAM on a GPU. In particular, the paper shows comparison of execution time of various lattice reduction aided detectors on a GPU. The results fulfill the requirements for WiMAX. Ahn, Bang, Kim, Lee, Kim, Choi, and Glossner consider SDR systems based on GPU cluster. The authors have implemented WiMAX and LTE on GPUS and analyzed both down link and up link performance. The results show that a cluster consisting of three GPUs can provide speedup of 2.5 compared to a single GPU system. LDPC decoding on general-purpose graphics processing units is topic of Grönroos, Nybom, and Björkqvist. The authors have avoided the memory bottleneck due to irregular memory accesses by using appropriate data structures for log-domain decoding. The authors have used LDPC codes specified in DVB standards as an example and show that high-performance decoding can also be realized on modern SIMD-enable multicore CPUs.

Two articles consider design methods. Guenther, Kempf, Ishaque, and Ascheid describe implementation of SDR systems on multiprocessor system-on-chip with the aid of Nucleus methodology. The paper discusses implementation of MIMO OFDM transceiver with timing specifications from IEEE $802.11 \mathrm{~m}$ standard. The authors have identified the kernels in the application and mapped them on a multicore platform. The experiment shows that the Nucleus approach speeds up development phase and improves portability. Methods for describing waveforms in SDR systems are considered by Dickens and Laneman. 
They propose a text-based algebraic language interface based on extensions in $\mathrm{C}++$, which simplifies the programming abstraction without additional run-time overhead.

Finally testing and measurement are discussed in three articles. Dietrich, Wolfe, and Vanhoy discuss performance evaluation of CRs. They propose psychometric models, especially item response models, reminding psychological models used for testing humans to improve the efficiency of CR testing. The authors have carried out simulations, which illustrate the potential of the proposed methods. Amanna, Ali, Fitch, and Reef discuss testing of SDR systems. The objective is to find interaction with system parameters and system performance. They propose a systematic approach, which provides tools for testing, identification of initialization parameters, and learning representative information about the system. Pitkänen, Jamieson, Becker, Moisio, and Takala discuss power consumption analysis of reconfigurable platforms. The paper introduces GroundHog 2009 benchmark suite and describes a method to generate test benches for power consumption measurement for the benchmark. Several configurable platforms are analyzed to illustrate the applicability of the benchmark.

As guest editors of this special issue, we sincerely thank the authors for their valuable contributions and all the anonymous reviewers for their feedback and help in ensuring a high level of quality. We also extend our appreciation to Janani Kalidasan and S. Shenbagam for their help on setting up this issue. We hope that you enjoy the special issue and find the articles informative and useful.

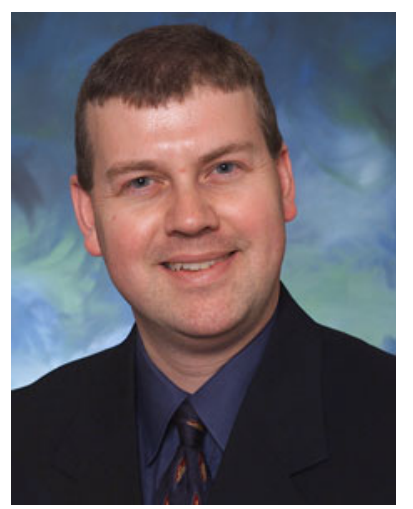

John Glossner is CMO and EVP of Wuxi DSP China and CEO of optimum semiconductor technologies, the US division of Wuxi DSP. Prior to joining Wuxi DSP John co-founded Sandbridge Technologies and served as EVP and CTO. Prior to Sandbridge, John managed both technical and business activities in DSP and broadband communications at IBM and Lucent/Starcore. John received a $\mathrm{Ph} . \mathrm{D}$. in Computer Architecture from TU Delft in the Netherlands, M.S. degrees in EE and Eng Mgt from NTU, and a BSEE degree from Penn State. John is a member of the Board of Directors and Chair of the Commercial Baseband Working Group of the Wireless Innovation Forum, a Senior member of the IEEE and has more than 120 publications and 36 issued patents.

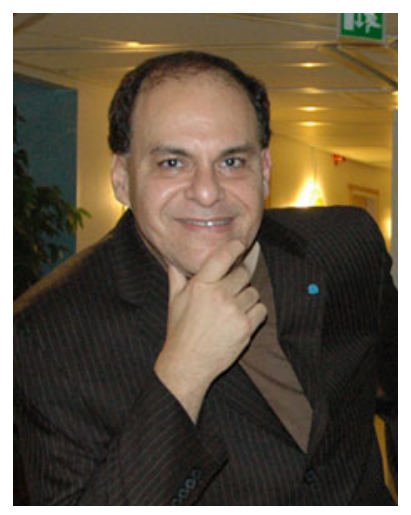

Mohammed Ismail joined Khalifa University of Science, Technology and Research (KUSTAR), UAE in April 2011. He spent over 25 years in academia and industry in the US and Europe and is the Founder of the Ohio State University's (OSU) Analog VLSI Lab, one of the foremost research entities in the field of analog, mixed signal and RF integrated circuits. He also served on the Faculty of OSU's ElectroScience Lab. He held a Research Chair at the Swedish Royal Institute of Technology (KTH) where he founded the RaMSiS (Radio and Mixed Signal Integrated Systems) Research Group there. He had visiting appointments in Finland (Aalto university), Norway (NTH and University of Oslo), the Netherlands (Twente University) and Japan (Tokyo Institute of Technology). At KUSTAR, Dr. Ismail holds the ATIC Professor Chair and serves as Director of the Sharjah Campus. He is the Founding Chair of the newly established ECE Department which exists on both campuses (Sharjah and Abu Dhabi) of the University and has its roots in the Etisalat College established in 1989. It now has over 40 faculty and 500 students, 450 undergraduate and 50 graduate students pursuing MS and PhD programs. Dr. Ismail has initiated the new KUSTAR's ICT Research Center encompassing 5 Research Labs conducting multidisciplinary research in information security, e-services and networks, multimedia communications and embedded mixed signal systems. He is working on creating the ATIC-KUSTAR Semiconductor Research Center focusing on low power System on Chip (SoC) design, test and IP development targeting the strategic areas of low power wireless chip sets, bio chips, self powered devices, power managements as well as research in the emerging fields of nanoscience. The Research is aligned with the 2030 Abu Dhabi strategic plan calling for diversification of the economy beyond oil and gas and for promoting innovation, entrepreneurship and spinoffs in the semiconductor, energy and ICT sectors among others. Dr. Ismail's current research focuses on robust low power RF and mm-wave ICs for wireless, bio and multimedia applications with a focus on manufacturable low cost high volume nanometer CMOS solutions for mobile and wearable embedded devices. A more recent interest is on nanoscience aiming to develop devices in the sub 10 nanometer range based on nano Schottky contacts. He served as a Corporate Consultant to over 30 companies and is a Co-Founder of Micrys Inc., Columbus, Ohio, Spirea AB, Stockholm, Firstpass Technologies Inc., Dublin, Ohio and ANACAD-Egypt (now part of Mentor Graphics). He advised the work of over $50 \mathrm{Ph} . \mathrm{D}$. students and of over 100 M.S. students. He authored or co-authored over a dozen books and over 150 journal publications and has 11 US patents. He is the Founding Editor of the Springer Journal of Analog Integrated Circuits and Signal Processing and serves as the Journal's Editor-inChief. He served the IEEE in many editorial and administrative capacities. He is the Founder of the IEEE International Conference on Electronics, Circuits and Systems (ICECS), the flagship Region 8 Conference of the IEEE Circuits and Systems Society. He is a Consulting Editor of the Springer Advanced Analog Book Series. He received the US Presidential Young Investigator Award, the Ohio State Lumley Research Award four times, in 1992, 1997, 2002 and 2007 and the US Semiconductor Research Corporation's Inventor Recognition Award twice. He is a Fellow of IEEE. 


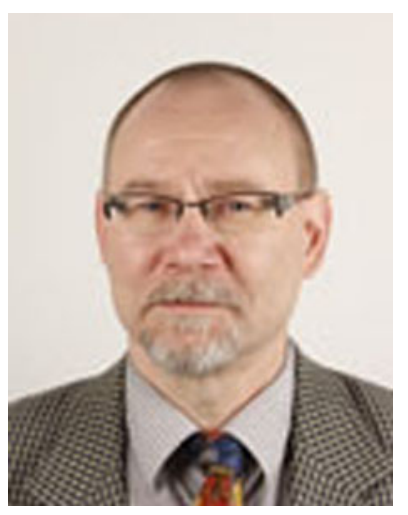

Jarmo Takala received his M.Sc. (hons) degree in Electrical Engineering and Dr. Tech degree in Information Technology from Tampere University of Technology, Tampere, Finland (TUT) in 1987 and 1999, respectively. From 1992 to 1995, he was a Research Scientist at VTT-Automation, Tampere, Finland. Between 1995 and 1996, he was a Senior Research Engineer at Nokia Research Center, Tampere, Finland. From 1996 to 1999, he was a Researcher at TUT. Currently, he is Professor in Computer
Engineering at TUT and Head of the Department of Computer Systems of TUT. His research interests include circuit techniques, parallel architectures, and design methodologies for digital signal processing systems. 